\title{
Comparison of human papillomavirus genotypes in high-grade squamous intraepithelial lesions and invasive cervical carcinoma: evidence for differences in biologic potential of precursor lesions
}

\author{
Rosemary E Zuna ${ }^{1}$, Richard A Allen ${ }^{1}$, William E Moore ${ }^{2}$, Rubina Mattu ${ }^{1}$ and \\ S Terence Dunn ${ }^{1}$ \\ ${ }^{1}$ Department of Pathology and ${ }^{2}$ Center for American Indian Health Research, University of Oklahoma Health \\ Sciences Center, Oklahoma City, OK, USA
}

\begin{abstract}
High-grade squamous intraepithelial lesions of the cervix are heterogeneous in their invasive potential. Comparison of human papillomavirus types between invasive cervical carcinoma and high-grade squamous dysplasia may provide insight into this biological variability. Liquid-based Pap specimens from $\mathbf{5 5}$ high-grade intraepithelial lesions and $\mathbf{4 7}$ invasive cervical carcinomas were analyzed by reverse line blot for $\mathbf{2 7}$ human papillomavirus types designated high, intermediate, or low risk. Human papillomavirus DNA was present in all high-grade intraepithelial lesions (23 different types) and in $94 \%$ (13 types) of invasive carcinomas. High-risk types were present in $\mathbf{8 1} \%$ of invasive carcinomas compared to $58 \%$ of high-grade intraepithelial lesions. Severe dysplasias harbored more $(\mathbf{7 9} \%)$ high-risk human papillomaviruses as compared to moderate dysplasias (37\%). In $40 \%$ of high-grade dysplasia cases (59\% of moderate dysplasias; $21 \%$ of severe) and $13 \%$ of invasive carcinomas, intermediate-risk genotypes were identified in the absence of high-risk HPV types. Human papillomavirus 16 was the most common type in all categories, including $47 \%$ of high-grade squamous dysplasias $(26 \%$ moderate; $68 \%$ severe) and $61 \%$ of invasive carcinomas. Both high-risk type $(P=0.0004)$ and type $16(P=0.0007)$ human papillomaviruses were positively associated with increasing severity of diagnosis. The heterogeneous nature of high-grade squamous dysplasias as compared to invasive carcinoma is evident by the wider spectrum of associated human papillomavirus types. Likewise, moderate dysplasia appears to be more heterogeneous in viral type than severe dysplasia. Moderate cases were more often associated with intermediate-risk types, while high-risk types were more prevalent in severe dysplasias and invasive cancers. Moderate dysplasia cases harboring viral types infrequently found in cancers may have a low risk for progression. Human papillomavirus genotyping of high-grade squamous intraepithelial lesions may be important in assessing risk for progression to invasion.

Modern Pathology (2004) 17, 1314-1322, advance online publication, 16 July 2004; doi:10.1038/modpathol.3800223
\end{abstract}

Keywords: cervical carcinoma; human papillomavirus; high-grade squamous intraepithelial lesion; HSIL; HPV genotyping

While the close association between human papillomavirus (HPV) infection and cervical neoplasia is

Correspondence: RE Zuna, MD, Department of Pathology, University of Oklahoma Health Sciences Center, 940 Stanton L Young Blvd., Biomedical Sciences Building Rm 451, Oklahoma City, OK 73104, USA.

E-mail: rosemary-zuna@ouhsc.edu

Presented in part at the 92nd Annual Meeting of the United States and Canadian Academy of Pathology in Washington, DC, March 22-29, 2003.

Received 9 January 2004; revised 28 May 2004; accepted 30 May 2004; published online 16 July 2004 well established, ${ }^{1-10}$ there is a wide disparity between the prevalence of infection and the occurrence of actual neoplasia. It is accepted that a small minority of women who are infected with HPV will develop high-grade intraepithelial lesions and still fewer will develop fully evolved carcinoma. In a recent study based in the US, 39\% of women aged between 18 and 40 years harbored at least one HPV type, including $36 \%$ with a negative Pap smear. ${ }^{11} \mathrm{In}$ the same study, $0.4 \%$ carried a cytologic interpretation of high-grade squamous intraepithelial lesion (HSIL). Thus, while HPV undeniably plays a role in 
the development of most cervical cancers, ${ }^{6,9,12,13}$ the reasons for the disparity between the high rates of HPV infection and prevalence of cervical neoplasia are not fully understood.

This disparity is reflected in the traditional pathology literature. In a meta-analysis of existing reports studying the natural history of cervical intraepithelial lesions, Östör ${ }^{14}$ estimated that severe dysplasia (CIN III) - the acknowledged immediate precursor lesion to invasive cervical carcinomaregresses $32 \%$ of the time, persists in $56 \%$ of cases, and progresses to invasion in $12 \%$ of affected women. Lesser grades of dysplasia show comparable variability with greater rates of regression. The possible reasons for this heterogeneity in biological outcome include host factors such as genetic factors, immune status, exposure to cocarcinogens, variability in host-viral interactions, and differences in HPV type.

In this report, we address the question of heterogeneity of HPV type as a possible source of biological variation ${ }^{2,15,16}$ in HSIL. This study compares HPV types in cases of HSIL and invasive cervical carcinoma using a highly sensitive polymerase chain reaction (PCR)-based reverse line-blot method (Roche HPV consensus PCR and genotyping tes $\mathrm{t}^{17,18}$ ) that simultaneously identifies one or more of 27 HPV types. The advantage of this approach is that only one amplification reaction is needed to identify a wide variety of HPV types associated with cervical disease. This gives a much more complete assessment of the variety of HPV types present in a clinical sample.

\section{Materials and methods}

\section{Patient Samples}

This study was approved by the Institutional Review Board at the University of Oklahoma Health Sciences Center. Patient samples were accumulated in two ways. Firstly, ThinPrep ${ }^{\circledR}$ Pap tests from OU Medical Center Cytopathology service with cytologic interpretations of HSIL were identified after sign-out. Prior to the date of regular discard of these residual samples, they were sent to the Molecular Pathology Laboratory for HPV testing by Hybrid Capture II (Digene Corporation, Gaithersburg, MD, USA) as part of our quality assurance monitoring program for cytopathology interpretations. ${ }^{19}$ In addition, residual HSIL samples were subjected to HPV genotyping using the Roche reverse line-blot method. A second source of specimens included hysterectomy and conization specimens from women undergoing therapy for the diagnosis of HSIL or invasive carcinoma. These were received fresh in the Surgical Pathology Laboratory. The lesion was scraped with a Cytobrush ${ }^{\circledR}$ (MedScand AB, Malmö, Sweden, USA) which was immediately immersed in PreservCyt $^{\mathbb{R}}$ (Cytyc Corp., Boxborough, MA, USA) and vigorously agitated against the side of the vial in order to dislodge cells. A ThinPrep ${ }^{\mathbb{B}}$ Pap slide was made from each PreservCyt ${ }^{\circledR}$ sample and the residual volume was then sent for Roche reverse line-blot HPV studies. ThinPrep ${ }^{\circledR}$ slides were examined in order to confirm the presence of diagnostic cells in each sample used for HPV genotyping. All HPV testing was performed without knowledge of cytologic or histologic interpretation.

\section{Cytologic and Histologic Diagnosis}

Cases were entered into the study on the basis of the original sign-out interpretation of HSIL (moderate or severe dysplasia) as part of the regular diagnostic cytopathology service, or of the histologic diagnosis of invasive cervical carcinoma. Owing to possible interobserver variation in the interpretation of cytologic specimens, ThinPrep ${ }^{\circledR}$ slides from the intraepithelial samples were masked and re-evaluated independently by two pathologists (RM and RZ) who were blinded to HPV type. Negative and low-grade squamous intraepithelial (LSIL) cases were also included to eliminate bias. The cytology slides were categorized on the basis of the most abnormal cell type identified according to the Bethesda System. ${ }^{20,21}$ In our laboratory, HSIL is characterized by dysplastic cells with dense, round-oval cytoplasm and increased nuclear-cytoplasmic ratio. In general, the cytoplasmic characteristics of HSIL are those of metaplastic cells, except in keratinizing dysplasias in which the categorization is based upon nuclear/cytoplasmic ratio. In severe dysplasia (HSIL-S), the cell size is small (comparable to parabasal cells) and the nuclearcytoplasmic ratio is exaggerated with the nucleus occupying greater than half of the cytoplasmic diameter. HSIL cells with larger amounts of dense cytoplasm are categorized as moderate dysplasia (HSIL-M). The cytologic categories were assigned values from 1 to 5 as follows: $1=$ Negative; $2=$ ASCUS (atypical cell of undetermined significance); $3=$ Mild dysplasia (CIN I, LSIL); $4=$ Moderate dysplasia (CIN II; HSIL-M); $5=$ Severe Severe dysplasia/carcinoma in situ (CIN III; HSIL-S). When mixed SIL patterns were identified, the cases were categorized by the most severe cell type identified. The scores of the two independent pathologists and the corresponding score of the original clinical interpretation made by one of four rotating diagnostic cytopathologists were summed for each case to derive a final score that represented a consensus diagnosis. Final scores of 3-4 were considered to be negative; 5-7, atypical (ASCUS); 810, mild dysplasia (LSIL); 11-13, moderate dysplasia (HSIL-M); and 14-15, severe dysplasia/carcinoma in situ (HSIL-S). For the 55 HSIL patients, the mean age was 34 years (median 32; range 16-78). For the 27 HSIL-M patients, the mean age was 32 years (median 29; range 16-78) while the mean age 
for the 28 HSIL-S women was 35 years (median 34; range 20-66).

The histologic diagnosis and cell type of each of 47 cases of invasive cervical carcinoma were originally made by a pathologist on the OU Medical Center diagnostic surgical pathology service, independently verified (RZ) without knowledge of the HPV type, and categorized according to the WHO classification. $^{22}$ There were 38 squamous cell carcinomas, four adenocarcinomas, three adenosquamous carcinomas, and two poorly-differentiated carcinomas. For the 47 carcinoma patients, the mean age was 41 years (median 39; range 24-76). The age distribution for women in the study is shown in Figure 1.

\section{HPV Genotyping}

A total amount of $5 \mathrm{ml}$ of residual PreservCyt ${ }^{\mathbb{R}}$ was aliquoted into individual $15 \mathrm{ml}$ tubes and were centrifuged at $2900 \mathrm{~g}$ for $5 \mathrm{~min}$. The supernatant was then removed and discarded and the cell pellet was resuspended in $200 \mu$ l of lysis buffer containing $20 \mu \mathrm{l}$ protease (QiaAmp DNA Blood Mini Kit, Qiagen, Valencia, CA, USA) and DNA was extracted following the manufacturer's instructions. For each specimen, $10 \mu \mathrm{l}$ of extracted DNA was subject to PCR amplification using the HPV consensus PCR and genotyping method (Roche Molecular Systems, Inc., Alameda, CA, USA), as indicated by the manufacturer. This method ${ }^{17,18}$ utilizes biotinylated primers (PGMY09/PGMY11) that amplify a $450 \mathrm{bp}$ fragment of the L1 open-reading frame of a broad spectrum of genital HPV genotypes. A control set of biotinylated primers (BGH20/BPC04) simultaneously amplifies a $268 \mathrm{bp}$ fragment of the human $\beta$-globin gene in each reaction. Resulting PCR products were then denatured and hybridized to separate HPV genotyping strips containing an immobilized array of 27 different HPV probes. These probes were categorized as high risk: $16,18,31,45$; intermediate risk:

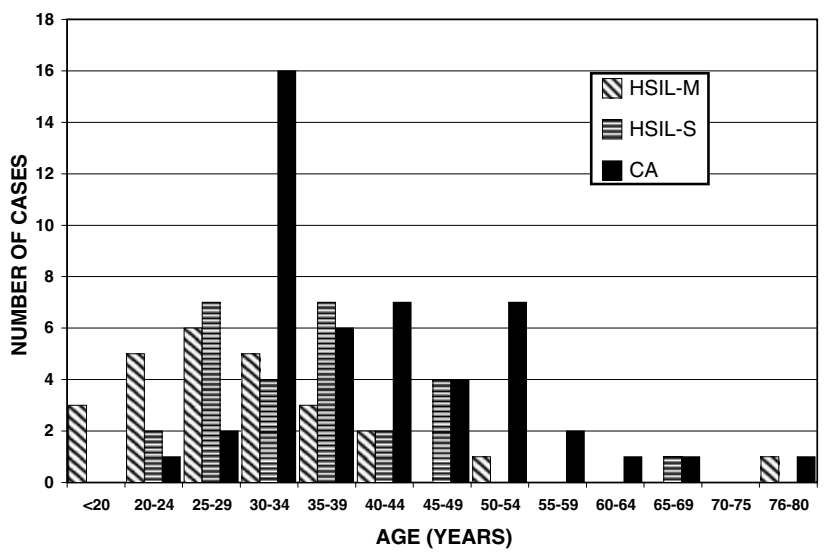

Figure 1 Age distribution of cases with HSIL-M (moderate dysplasia), HSIL-S (severe dysplasia) and invasive carcinoma (CA).
33, 35, 39, 51, 52, 56, 58, 59, 68 (ME180), 73 (MM9, P238A), 82 (MM4, W13B), 83 (MM7, P291); and low risk: 6, 11, 26, 40, 42, 53, 54, 55, 57, 66, 84 (MM8, P155). Hybridized PCR products were visualized by using a streptavidin-horseradish peroxidase detection system. Each genotyping strip also contains $\beta$-globin probes at two different concentrations to control for interpretation of sample inhibition and sufficiency. Samples displaying hybridization to only the high concentration $\beta$-globin probe (indicative of either PCR inhibition or extremely low cell count) were discounted from final analysis. A negative control was run with each batch of specimens tested and was consistently negative.

\section{Statistical Analyses}

Statistical analyses were performed using the Systat Version 10 (Systat Software Inc., Richmond, CA, USA) and StatXact 5 (Cytel Software Inc., Cambridge, MA, USA). Inferential statistics used for tabular data included Fisher's exact tests, Pearson $\chi^{2}$, odds ratios with $95 \%$ confidence intervals, and Cochran-Armitage linear trend tests. All $P$-values were two-sided. Statistical significance was ascribed to $P$-values $\leq 0.05$.

\section{Results}

The distribution of HPV types found in the 55 HSIL and 47 invasive carcinoma cases is shown in Table 1. A comparison of the age to cytologic category is shown in Figure 1. All of the 55 HSIL cases and 44 of $47(94 \%)$ cancer cases contained detectable HPV DNA. The pattern of HPV types identified in our cases of invasive carcinoma is comparable to that described worldwide. ${ }^{10,12,13}$ Overall, 23 different HPV types were found in the 55 cases of HSIL compared with 13 different types in the 47 invasive carcinoma cases. Multiple HPV types were found more frequently in individual HSIL $(22 / 55 ; 40 \%)$ than in invasive carcinoma $(8 / 47 ; 17 \%)$ cases (odds ratio (OR), 3.25, 95\% confidence interval (CI); 1.3-8.3)

Overall, $38(81 \%)$ of carcinomas harbored highrisk viral types compared with 32 (58\%) of HSIL cases (OR, 3.04, 95\% CI; 1.2-7.5). Categorization of HSIL into HSIL-M and HSIL-S, revealed significant differences in the occurrence of HPV types; $77 \%$ of HSIL-S cases harbored HPV 16 compared with only $37 \%$ of HSIL-M (OR, 6.23, 95\% CI; 1.9-20.6). Highrisk type $(P=0.0004)$ and HPV $16(P=0.0007)$ were positively associated with increasing severity of diagnosis when examined across the ordered diagnostic categories of HSIL-M, HSIL-S and invasive carcinoma using the Cochran-Armitage trend test. While there was no difference in the occurrence of high-risk HPV between HSIL-S and invasive carcinoma (OR, 1.15, 95\% CI; 0.4-3.6), high-risk types were significantly less frequent in our cases of HSIL-M 
Table 1 Distribution of HPV types in HSIL and invasive cervical carcinoma

\begin{tabular}{|c|c|c|c|c|c|}
\hline \multirow{2}{*}{$\begin{array}{l}\text { HPV } \\
\text { category }\end{array}$} & \multirow{2}{*}{$\begin{array}{l}H P V \\
\text { type }\end{array}$} & \multicolumn{3}{|c|}{ High-grade SIL } & \multirow{2}{*}{$\begin{array}{l}\text { Carcinoma } \\
\mathrm{n}(\%)^{\mathrm{a}}\end{array}$} \\
\hline & & $\begin{array}{l}H S I L-M \\
\mathrm{n}(\%)^{\mathrm{a}}\end{array}$ & $\begin{array}{l}H S I L-S \\
\mathrm{n}(\%)^{\mathrm{a}}\end{array}$ & $\begin{array}{l}\text { Total HSIL } \\
\text { n }(\%)^{\mathrm{a}}\end{array}$ & \\
\hline \multirow[t]{4}{*}{ High risk } & 16 & $7(26)^{\mathrm{b}}$ & $19(68)^{\mathrm{b}}$ & $26(47)^{\mathrm{b}}$ & $29(62)^{\mathrm{b}}$ \\
\hline & 18 & $2(7)^{b}$ & $2(7)^{\mathrm{c}}$ & $4(7)^{b}$ & $10(21)^{\mathrm{b}}$ \\
\hline & 31 & $1(4)^{\mathrm{c}}$ & $2(7)^{\mathrm{b}}$ & $3(5)^{b}$ & 0 \\
\hline & 45 & $1(4)^{\mathrm{c}}$ & 0 & $1(2)^{\mathrm{c}}$ & $1(2)^{b}$ \\
\hline \multirow{12}{*}{$\begin{array}{l}\text { Intermediate } \\
\text { risk }\end{array}$} & 33 & $1(4)^{\mathrm{c}}$ & $1(4)^{\mathrm{b}}$ & $2(4)^{\mathrm{b}}$ & $3(6)^{b}$ \\
\hline & 35 & $6(22)^{\mathrm{b}}$ & $2(7)^{\mathrm{b}}$ & $8(15)^{\mathrm{b}}$ & $1(2)^{b}$ \\
\hline & 39 & $4(15)^{\mathrm{b}}$ & $2(7)^{\mathrm{c}}$ & $6(11)^{\mathrm{b}}$ & 0 \\
\hline & 51 & $5(19)^{b}$ & $1(4)^{\mathrm{c}}$ & $6(11)^{\mathrm{b}}$ & 0 \\
\hline & 52 & $4(15)^{b}$ & $2(7)^{\mathrm{c}}$ & $6(11)^{b}$ & 0 \\
\hline & 56 & $2(7)^{\mathrm{b}}$ & $3(11)^{\mathrm{c}}$ & $5(9)^{b}$ & $1(2)^{\mathrm{c}}$ \\
\hline & 58 & $1(4)^{\mathrm{c}}$ & 0 & $1(2)^{\mathrm{c}}$ & 0 \\
\hline & 59 & $4(15)^{b}$ & $1(4)^{\mathrm{c}}$ & $5(9)^{b}$ & $1(2)^{\mathrm{c}}$ \\
\hline & 68 & 0 & $1(4)^{\mathrm{c}}$ & $1(2)^{\mathrm{c}}$ & 0 \\
\hline & 73 & 0 & $1(4)^{\mathrm{c}}$ & $1(2)^{\mathrm{c}}$ & $1(2)^{\mathrm{c}}$ \\
\hline & 82 & 0 & 0 & 0 & $1(2)^{\mathrm{c}}$ \\
\hline & 83 & $1(4)^{\mathrm{c}}$ & 0 & $1(2)^{\mathrm{c}}$ & $2(4)^{b}$ \\
\hline \multirow[t]{12}{*}{ Low risk } & 6 & $1(4)^{\mathrm{c}}$ & 0 & $1(2)^{\mathrm{c}}$ & 0 \\
\hline & 11 & 0 & 0 & 0 & 0 \\
\hline & 26 & 0 & $1(4)^{\mathrm{c}}$ & $1(2)^{\mathrm{c}}$ & 0 \\
\hline & 40 & 0 & 0 & 0 & 0 \\
\hline & 42 & 0 & $1(4)^{\mathrm{c}}$ & $1(2)^{\mathrm{c}}$ & 0 \\
\hline & 43 & 0 & 0 & 0 & 0 \\
\hline & 53 & $2(7)^{\mathrm{b}}$ & $1(4)^{\mathrm{c}}$ & $3(5)^{b}$ & $1(2)^{\mathrm{c}}$ \\
\hline & 54 & 0 & $1(4)^{\mathrm{c}}$ & $1(2)^{\mathrm{c}}$ & $1(2)^{\mathrm{c}}$ \\
\hline & 55 & $2(7)^{\mathrm{c}}$ & $1(4)^{\mathrm{c}}$ & $3(5)^{c}$ & $2(4)^{\mathrm{c}}$ \\
\hline & 57 & 0 & 0 & 0 & 0 \\
\hline & 66 & $1(4)^{\mathrm{C}}$ & $3(11)^{\mathrm{c}}$ & $4(7)^{\mathrm{c}}$ & 0 \\
\hline & 84 & 0 & $1(4)^{\mathrm{c}}$ & $1(2)^{\mathrm{c}}$ & 0 \\
\hline Negative & & 0 & 0 & 0 & $3(6)$ \\
\hline Total types & & 45 & 46 & 91 & 54 \\
\hline Total cases & & 27 & 28 & 55 & 47 \\
\hline
\end{tabular}

${ }^{\mathrm{a}}$ Since some cases contained more than one HPV type (Table 2), the total number of types present is greater than the number of cases. As a result, the columns do not add to $100 \%$.

${ }^{b_{T h}}$ This type was present as the sole HPV identified in at least one case. ${ }^{\mathrm{C}}$ This type was identified only as one type in a multiple infection case and may not be associated with the most significant lesion. when compared with the combined category of HSIL-S and invasive carcinoma (OR, 6.8, 95\% CI; 2.6-17.8).

While high-risk viral types were found less frequently in HSIL-M than HSIL-S and invasive carcinoma, intermediate-risk HPV types were more common in HSIL-M. These viral types were the highest risk type in $59 \%$ of HSIL-M as compared with $21 \%$ of HSIL-S and $13 \%$ of invasive cancers (Table 2). These results demonstrate a negative association between increasing diagnostic severity and intermediate-risk HPV frequency (CochranArmitage trend test, $P<0.0001$ ).

Although low-risk viral types were identified in 13 of $55(24 \%)$ HSIL cases and in three of $47(6 \%)$ carcinomas (Table 1), there was only one HSIL-M case (see below) in which a low-risk HPV type (type 53) was present as the sole HPV type (Table 2). Otherwise, low-risk types were identified in this population only in cases with multiple HPV infections in which higher risk viruses were also present.

HPV 16 was the most frequent viral type in the cancer cases (Tables 1 and 4), identified in $29(62 \%)$ cases. HPV 18 was identified in 10 cases $(21 \%)$ of which five were adenocarcinomas or adenosquamous carcinomas. HPV 33 was identified in three $(6 \%)$ cases and HPV 45 in one $(2 \%)$ case. Three cancer cases $(6 \%)$ were HPV negative using this technique. In two squamous cell carcinoma cases, both HPV 16 and HPV 18 were identified. Intermediate-risk viruses were also identified in nine cases. HPV 33, 35, and 83, listed here as intermediate risk, were identified as single infections in four $(9 \%)$ cancer cases. In the remaining five cases, the lesser risk viruses coexisted with high-risk types.

For HSIL cases (Table 1), the most frequent viral types were HPV 16 (47\% of cases), HPV 35 (15\%), HPV 39, 51, and 52 (11\% each) and HPV 56 and 59 ( $9 \%$ each). When HSIL was divided into HSIL-M (Figure 2) and HSIL-S (Figure 3), differences in the distribution of viruses within these cytologic

Table 2 Distribution of cervical lesions according to highest risk type

\begin{tabular}{|c|c|c|c|c|c|}
\hline \multirow[t]{2}{*}{ HPV category } & \multicolumn{4}{|c|}{ Diagnostic category } & \multirow[t]{2}{*}{ Total n $(\%)$} \\
\hline & $\begin{array}{c}H S I L-M \\
\mathrm{n}(\%)\end{array}$ & $\begin{array}{c}H S I L-S \\
\mathrm{n}(\%)\end{array}$ & $\begin{array}{l}\text { Total HSIL } \\
\text { n (\%) }\end{array}$ & $\begin{array}{c}\text { Carcinoma } \\
\text { n }(\%)\end{array}$ & \\
\hline High risk $^{\mathrm{a}}$ & $10(37)$ & $22(79)$ & $32(58)$ & $38(81)$ & $70(69)$ \\
\hline Intermediate risk ${ }^{\mathrm{b}}$ & $16(59)$ & $6(21)$ & $22(40)$ & 6 (13) & $28(27)$ \\
\hline Low risk ${ }^{\mathrm{c}}$ & $1(4)$ & 0 & $1(2)$ & 0 & $1(1)$ \\
\hline Negative & 0 & 0 & 0 & $3(6)$ & $3(3)$ \\
\hline Total cases & 27 & 28 & 55 & 47 & 102 \\
\hline
\end{tabular}

${ }^{\mathrm{a}}$ Includes HPV 16, 18, 31, and 45.

bIncludes HPV 33, 35, 39, 51, 52, 56, 58, 59, 68, 73, 82, and 83.

${ }^{\mathrm{c}}$ Includes HPV 6, 11, 26, 40, 42, 53, 54, 55, 57, 66, and 84 . 

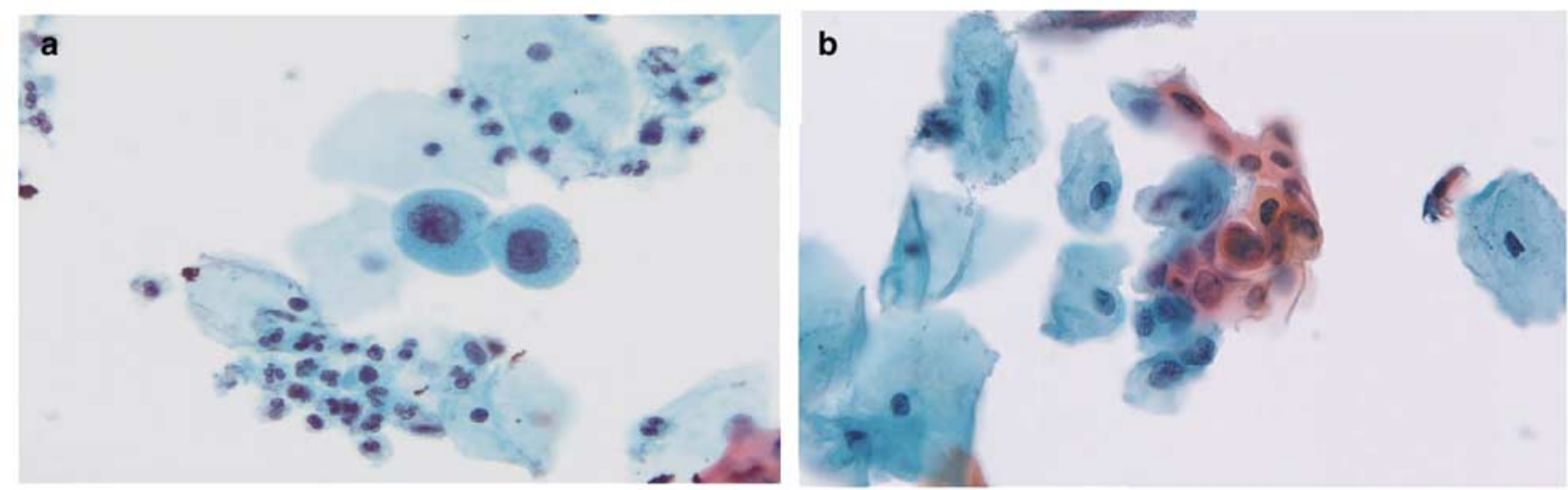

c

d

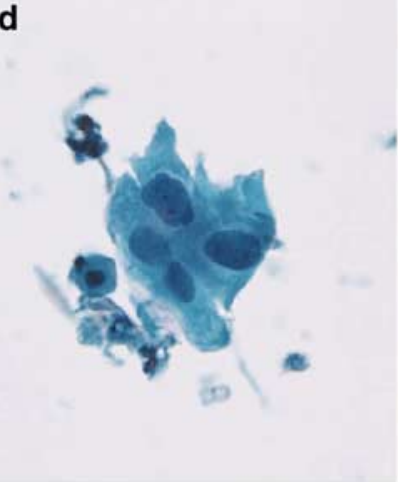

e

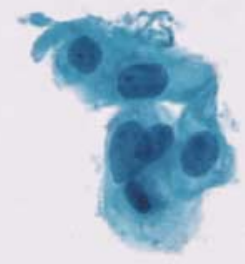

Figure 2 Cases of HSIL-M (moderate dysplasia) associated with a single HPV type (Papanicolaou stain, $\times 600$, original magnification): (a) HPV 18 in a 19-year-old woman; (b) HPV 35 in a 26-year-old woman; (c) HPV 56 in a 33-year-old woman; (d) and (e) HPV 52 in a 39-yearold woman.
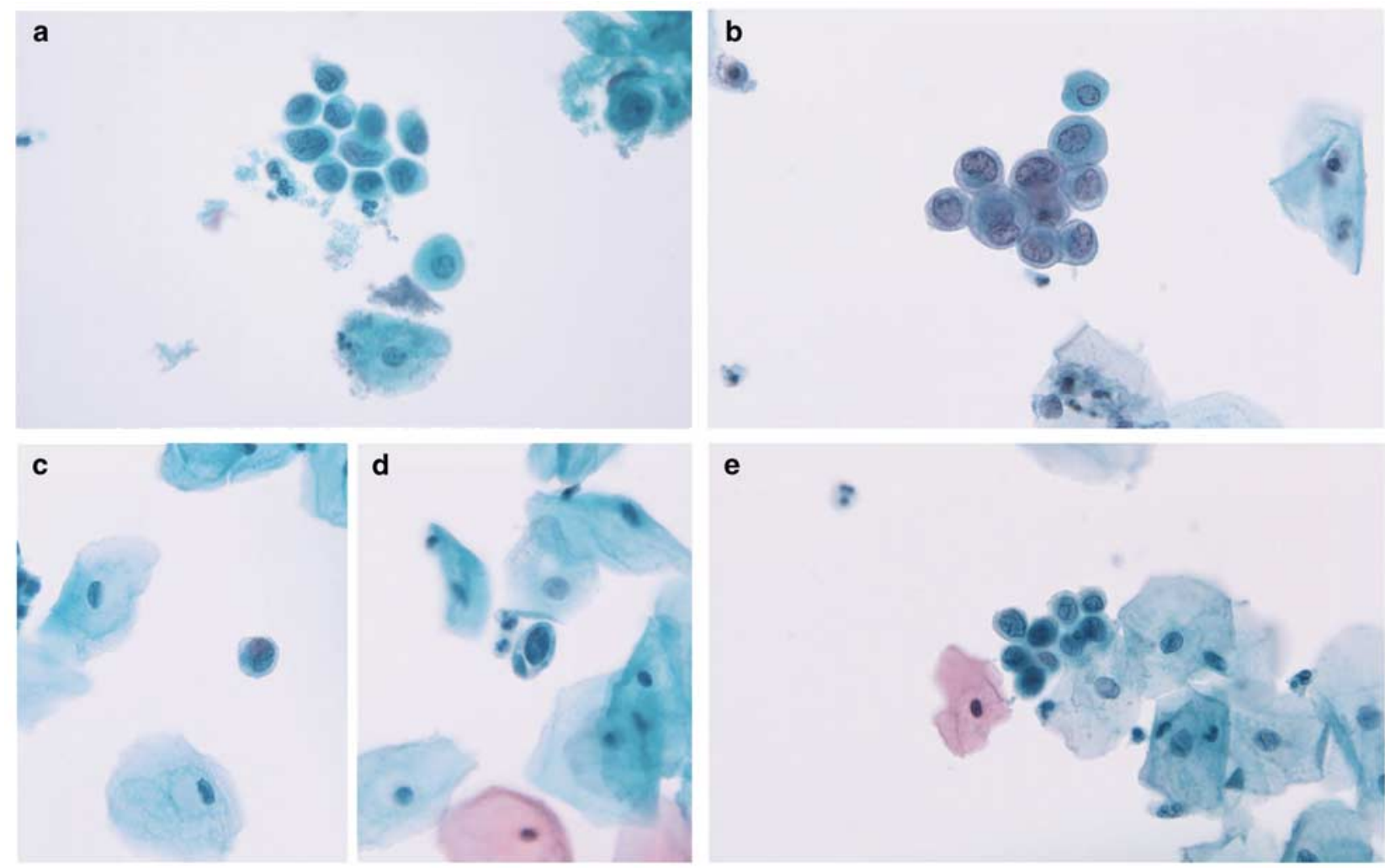

Figure 3 Cases of HSIL-S (severe dysplasia) associated with a single HPV type (Papanicolaou stain, $\times 600$, original magnification): (a) HPV 16 in a 32-year-old; (b) HPV 35 in a 38-year-old; (c) and (d) HPV 31 in a 35-year-old; (e) HPV 16 in a 32 -year-old. 
categories emerged. While HPV 16 was again the most frequent viral type for both categories, the relative percentage of cases harboring HPV 16 varied from $68 \%(19 / 27)$ for HSIL-S to $26 \%(7 / 28)$ for HSILM (OR, 6.03, 95\% CI; 1.9-19.4). The percentage of cases of HSIL-S that harbored HPV 16 closely resembled the pattern $(62 \%, 29 / 47)$ for carcinomas (OR, 1.31, 95\% CI; 0.5-3.5). The pattern of distribution of HPV 16 in HSIL and invasive carcinoma is further highlighted in Table 3 . While 53\% (25/47) of cancers and $46 \%(13 / 28)$ of HSIL-S contained HPV 16 as the only HPV type (OR, 1.31, 95\% CI; 0.5-3.44), HSIL-M differed with only 15\% (4/27) showing only HPV 16 infection. When invasive carcinoma and HSIL-S combined are compared to HSIL-M, it is apparent that they have a greater frequency of isolated HPV 16 infection (OR, 5.91, 95\% CI; 1.9-18.4). This pattern was maintained when cases that contained HPV16 as part of a mixed infection were added to the analysis.

HPV 18 was relatively under-represented in HSIL $(7 \% ; 4 / 55)$ in this series as compared to carcinomas $(21 \% ; 10 / 47)$. However, half of the HPV 18 positive carcinoma cases (Table 4) were either adenocarcinomas or adenosquamous carcinomas while no intraepithelial glandular lesions were identified in the HSIL cases.

The distribution of intermediate-risk types (HPV 35, 39, 51, 52, and 59) varied from 15 to $22 \%$ (Table 1) for HSIL-M, compared with $4-7 \%$ for HSIL-S. The relative distribution of intermediate-risk viruses was less frequent in the carcinomas $(0-2 \%)$ as compared to HSIL-S cases. HPV 53, listed here as low risk, was found as the sole HPV type in only one HSIL case, that of an HSIL-M lesion.

Table 3 Comparison of pattern of HPV types in HSIL and carcinoma

\begin{tabular}{|c|c|c|c|c|}
\hline \multirow[t]{2}{*}{ Pattern of HPV Types } & \multicolumn{3}{|c|}{ High-grade SIL } & \multirow{2}{*}{$\begin{array}{c}\text { Carcinom } \\
\text { n }(\%)\end{array}$} \\
\hline & $\begin{array}{c}\text { HSIL-M } \\
\text { n (\%) }\end{array}$ & $\begin{array}{c}H S I L-S \\
\mathrm{n}(\%)\end{array}$ & $\begin{array}{c}\text { Total HSIL } \\
\text { n (\%) }\end{array}$ & \\
\hline \multicolumn{5}{|l|}{ Number of HPV types/case } \\
\hline No HPV & 0 & 0 & 0 & $3(6)$ \\
\hline 1 & $16(59)$ & $17(61)$ & $33(60)$ & $36(77)$ \\
\hline 2 & 5 (19) & $6(21)$ & $11(20)$ & 7 (15) \\
\hline 3 & $5(19)$ & $4(14)$ & $9(17)$ & $1(2)$ \\
\hline$>3$ & $1(3)$ & $1(4)$ & $2(4)$ & 0 \\
\hline \multicolumn{5}{|l|}{ HPV risk categories/case } \\
\hline High-risk HPVª & $10(37)$ & $22(79)$ & $32(58)$ & $38(81)$ \\
\hline Intermediate-risk $\mathrm{HPV}^{\mathrm{a}}$ & $20(74)$ & $10(36)$ & $30(55)$ & 9 (19) \\
\hline Low-risk HPV ${ }^{\mathrm{a}}$ & $6(22)$ & 7 (25) & $13(24)$ & $3(6)$ \\
\hline One high-risk type onlyb & $5(19)$ & $15(54)$ & $20(36)$ & $36(75)$ \\
\hline HPV $16^{\mathrm{a}}$ & $7(26)$ & $19(68)$ & $26(47)$ & $29(62)$ \\
\hline HPV 16 only & 4 (15) & $13(46)$ & $17(31)$ & $25(53)$ \\
\hline No high-risk types ${ }^{\mathrm{a}}$ & $17(63)$ & $6(21)$ & $23(42)$ & $9(19)$ \\
\hline Total cases & 27 & 28 & 55 & 47 \\
\hline
\end{tabular}

${ }^{\mathrm{a}}$ Cases may contain multiple HPV types.

${ }^{\mathrm{b}}$ Cases containing only one HPV type that is high risk.

Table 4 Distribution of HPV 16 in cervical lesions according to diagnostic category

\begin{tabular}{|c|c|c|c|c|c|}
\hline Diagnosis & $\begin{array}{l}H P V 16 \text { only } \\
\mathrm{n}(\%)^{\mathrm{b}}\end{array}$ & $\begin{array}{c}H P V 16 \text { and other } H P V^{\mathrm{c}} \\
\mathrm{n}(\%)\end{array}$ & $\begin{array}{c}\text { Total HPV } 16+ \\
\mathrm{n}(\%)\end{array}$ & $\begin{array}{c}H P V+\text { but not } H P V 16^{\mathrm{d}} \\
\mathrm{n}(\%)\end{array}$ & Total cases \\
\hline HSIL-M & $4(15)$ & $3(11)$ & $7(26)$ & $20(74)$ & 27 \\
\hline HSIL-S & $13(46)$ & $6(21)$ & $19(68)$ & $9(32)$ & 28 \\
\hline All HSIL & $17(31)$ & 9 (16) & $26(47)$ & $29(53)$ & 55 \\
\hline Carcinoma $^{e}$ & $25(53)$ & $4(9)$ & $29(62)$ & $15(32)$ & $47^{\mathrm{f}}$ \\
\hline
\end{tabular}

${ }^{\mathrm{a}} \mathrm{HPV} 16$ present as single HPV type.

${ }^{b}$ Percentages calculated across the rows.

${ }^{\mathrm{c}} \mathrm{HPV} 16$ present as one of two or more HPV types.

${ }^{\mathrm{d}} \mathrm{HPV}$ positive but not HPV 16.

${ }^{\mathrm{e}}$ Includes adenocarcinomas and adenosquamous carcinomas.

${ }^{\mathrm{f}}$ Three carcinoma cases were negative for HPV. 


\section{Discussion}

The biologic heterogeneity of the so-called cervical cancer precursors is a fascinating scientific question with important clinical and economic implications. Identifying the key features that would allow reliable categorization of lesions into those relatively few cases at high risk for progression and the much larger number of cases with little such risk would allow strategic and targeted intervention for those patients at highest risk. At the same time, follow-up paradigms could be constructed for women with lesser risk lesions. Such paradigms would need to establish a safety net for identifying subsequent risk for progression while reducing costly and unnecessary therapeutic intervention for lesions that are destined to regress.

As an initial step in addressing this issue, we elected to determine the HPV types present in our cases of HSIL and to compare the distribution of these HPV types with those found in cases of invasive cervical cancer. HSIL cases that harbor HPV types that are identified as a single genotype in carcinomas would appear likely to be true precursors to cancer. Those HSIL cases that are associated with HPV types that are not seen in cancers or are present only in mixed infections may be lesions that would ultimately regress or persist unchanged.

While previous authors have reported HPV patterns for various intraepithelial subcategories, ${ }^{2,4,8,15,16,23}$ our study differs in that it directly compares HSIL and carcinoma using a technique that simultaneously tests for a relatively large number of HPV types. Although the distribution of HPV types in carcinoma and HSIL overlapped in this study, these results highlight a more complicated pattern of HPV types in HSIL lesions as compared with carcinoma.

These findings lend support to the suggestion that differences in HPV type may indeed correlate with differences in biological potential and risk for invasion in HSIL lesions. ${ }^{2,15,24}$ The viral genotypes most closely associated with invasive squamous cancer, particularly HPV 16 and related types, were those most closely associated with HSIL-S in this study. While this association of carcinoma and HSIL with high-risk HPV types has been well described in the literature, ${ }^{1-5}$ the finding that HSIL-M had a different, highly varied pattern of HPV genotypes has not been well recognized. Unlike invasive carcinoma and HSIL-S, most of the HPV types identified in HSIL-M were in the intermediate-risk category. These intermediate-risk types were rarely present as the sole HPV in our invasive cancers. This is significant because HSIL-M and HSIL-S are currently grouped into a single category for purposes for cytologic interpretation using Bethesda 2001 terminology ${ }^{25}$ and also for clinical follow-up regimens. ${ }^{26}$ It has previously been recognized ${ }^{14,27-29}$ that most cases of LSIL regress spontaneously, while HSIL cases, as a group, present a greater risk for progression. ${ }^{14}$ The results of this study suggest that subcategories exist within HSIL in which risk for progression to carcinoma is related, at least in part, to HPV type. These results are consistent with the meta-analysis published by Clifford et $a l^{24}$ comparing the HPV types in HSIL lesions and squamous cell carcinoma.

The large number of cases in our series harboring multiple HPV types adds a high level of complexity to interpreting these data. Reid et $a l^{30}$ found that individual colposcopic lesions harbor only one HPV type, suggesting that each HPV type identified in our samples represents an individual lesion. However, it is possible that some SIL lesions represent a fusion of individual HPV-associated lesions or even a coinfection of HPV types in a single clonal population. Our data cannot distinguish among these possibilities. However, invasive cancer cases showed a lesser number of multiple HPV types compared with HSIL suggesting that a limited number of HPV types are associated with the transition to invasion. If younger women harbor multiple HPV types including at least one oncogenic type, presumably the lesions harboring the lower risk viral types remit over time, while the oncogenic process persists and advances both spatially and in severity of the associated lesion. It should be noted that the samples from the cancer cases were taken primarily from the tumor in the surgical pathology laboratory (although much of the uninvolved cervix was also sampled), while the HSIL cases were clinical screening tests in which the surface of the cervix was thoroughly sampled. It is conceivable that this difference in specimen collection is at least partially responsible for the difference in the number of viral types identified in the cancers and the HSIL cases.

HPV 18 appears to be under-represented in HSIL in our series when compared to carcinomas. It has been suggested that HPV $18^{2,31}$ may be associated with rapidly evolving lesions such that an HSIL intermediate has not often been identified. An alternative hypothesis is that HPV 18 lesions are preferentially glandular in histogenesis ${ }^{32}$ and thus are more likely to be associated with glandular precursor lesions (adenocarcinoma in situ (AIS)) rather than HSIL. Thus, the relative disparity between HPV 18 in HSIL and cancers in this series may relate to the presence of the adenocarcinomas and adenosquamous carcinomas. This may also explain the higher rate of HPV 16 positivity in HSIL-S compared with HPV 18.

These data are at variance with the findings of Lungu et al, ${ }^{33}$ who found no difference in HPV types between CIN 2 and CIN 3. There were significant methodological differences between that study and ours that make comparison difficult. In that study, PCR was performed on biopsy digests using a restricted number of probes, while our study uses a cytologic sample and an expanded probe set. Similarly, interobserver variability in interpretation 
of both cytologic ${ }^{34-36}$ and histologic changes $^{37,38}$ in the cervix is well documented in the literature. The findings of Matsukura and Sugase, ${ }^{8}$ using a larger set of HPV types, show a varying pattern of HPV types with category of CIN similar to that described here. We are now including LSIL cases in our accumulating study set in order to compare the pattern of HPV types in HSIL and LSIL cases. It will be interesting to see if the HPV types found in LSIL are similar to or different from those present in HSIL-M. Nonetheless, the statistical significance that our data show in discriminating HSIL-M and HSIL-S according to HPV type and the lack of such discrimination between HSIL-S and invasive cervical carcinoma suggests a high level of consistency in our approach.

Muñoz et $a l^{10}$ have recently published a study in which HPV genotyping data was pooled from 11 case-controlled studies of 1739 squamous cell carcinoma cases worldwide. It is of interest that carcinoma in situ (categorized here as HSIL-S) was included in the list of cancers reported by Muñoz. They suggested that HPV types that we have categorized as intermediate risk be included in the group of high-risk HPV types. These include HPV $33,35,39,51,52,56,58,59,68,73$, and 82 . They also raised the possibility that HPV types 26,53 , and 66 (listed here as low risk) should also be considered in that category. In our study, albeit with a limited number of cases, HPV 33, 35, and 83 (listed here as intermediate risk) were identified as the single type in individual invasive carcinoma cases and thus may deserve consideration for recategorization as high risk. However, the remaining intermediate-risk viral types were present in our population predominantly in intraepithelial lesions and only as a part of mixed infections with higher risk types if present in the cancers.

Classically, CIN I (the histological equivalent of LSIL), CIN II (HSIL-M) and CIN III (HSIL-S) have been thought to represent a morphologic and biological progression of a single preneoplastic continuum that evolves over many years. Recent authors ${ }^{27-29}$ have suggested that LSIL (CIN I) and HSIL (CIN II and CIN III) represent two distinct categories with differing potential for progression such that LSIL lesions generally remit spontaneously. Our work here suggests that many HSIL lesions (HSIL-M (CIN II), in particular) are associated with HPV types that are not commonly found as the sole HPV type in cancers. It would seem then that invasive cancer would be an unlikely end point for such intraepithelial lesions. It is unclear at this time whether the viral types identified here as intermediate risk and found predominantly in HSIL-M cases are associated with lesions that ultimately regress like LSIL, or if such lesions have an intermediate potential. It is conceivable that intermediate risk-associated lesions could represent an incomplete form of viral/neoplastic transformation (recognized morphologically as dysplasia) that persists unchanged for extended periods.
On the other hand, it is possible that some lower grade lesions associated with oncogenic HPV types (especially HPV 16) may indeed undergo the classical progression from CIN I to invasive cancer. If so, HPV type would be an important parameter in assigning risk for progression in women with lowgrade lesions. If verified by additional studies, these observations can have significant implications for HPV testing and cervical cancer screening. If only a few viral types are truly associated with the fully evolved malignant phenotype, then it may be appropriate to identify these high-risk viruses in clinical HPV testing as a way of stratifying risk and, perhaps, directing therapeutic intervention. Thus, a combination of morphology and HPV genotyping would appear to be a powerful combination to stratify risk for an individual patient. Similarly, a smaller set of truly oncogenic viral types would simplify creation of HPV vaccines. We hypothesize that HPV type is an important variable that helps to stratify risk for progression to invasion in women with cervical dysplasia.

\section{Acknowledgements}

We acknowledge the assistance of Drs Michael Gold, Scott McMeekin, Joan Walker, Natalie Gould, Todd Tillmans, Teresa Rutledge, Barbara Bane, Zoltan Laszik, Gregory Blakey, Chimen Kesserwan, Adeboye Osunkoya, Ildiko Nagy, Hans Iwenofu, W Grant Davis, Pablo Souza, Fred Bierbaum, Robyn Potts, Jane Zhou, You $\mathrm{Lu}$, and Ravindranauth Sawh for their assistance in acquiring samples for this study. We also thank Cora Wright, Patricia Parrish and Danita Young in the Cytopathology Laboratory of OU Medical Center for their enthusiastic help in specimen handling. In addition, we are grateful to Roche Molecular Systems (Alameda, CA, USA) for the gift of the HPV consensus PCR and genotyping reagents used in this study.

\section{References}

1 zur Hausen H. Human papilloma viruses and their possible role in squamous cell carcinomas. Curr Top Micobiol Immunol 1977;78:1-3.

2 Lorincz A, Temple GF, Kurman RJ, et al. Oncogenic association of specific human papillomavirus types in cervical neoplasia. J Natl Cancer Inst 1987;79:671-677.

3 Morrison EAB, Ho GYF, Vermund SH, et al. Human papillomavirus infection and other risk factors for cervical neoplasia: a case control study. Int J Cancer 1991;49:6-13.

4 Lorincz A, Reid R, Jenson AB, et al. Human papillomavirus infection of the cervix: relative risk associations of 15 common anogenital types. Obstet Gynecol 1992;79:328-337.

5 Schiffman MH, Bauer HM, Hoover RN, et al. Epidemiologic evidence showing that human papilloma- 
virus infection causes most cervical intraepithelial neoplasia. J Natl Cancer Inst 1993;85:958-964.

6 Liaw K-L, Glass AG, Manos MM, et al. Detection of human papillomavirus DNA in cytologically normal women and subsequent cervical squamous intraepithelial lesions. J Natl Cancer Inst 1999;91:954-960.

7 Walboomers JMM, Jacobs MV, Manos MM, et al. Human papillomavirus is a necessary cause of invasive cervical cancer worldwide. J Pathol 1999;189:12-19.

8 Matsukura T, Sugase M. Relationship between 80 human papillomavirus genotypes and different grades of cervical intraepithelial neoplasia: association and causality. Virol 2001;283:139-147.

9 Bosch FX, Lorincz A, Muñoz N, et al. The causal relation between human papillomavirus and cervical cancer. J Clin Pathol 2002;55:244-265.

10 Muñoz N, Bosch FX, de Sanjosé S, et al. International Agency for Research on Cancer Multicenter Study Group. Epidemiologic classification of human papillomavirus types associated with cervical cancer. N Engl J Med 2003;348:518-527.

11 Peyton CL, Gravitt PE, Hunt WC, et al. Determinants of genital human papillomavirus detection in a US population. J Infect Dis 2001;183:1554-1564.

12 Bosch FX, Manos MM, Muñoz N, et al. Prevalence of human papillomavirus in cervical cancer: a worldwide perspective. J Natl Cancer Inst 1995;87:796-802.

13 Clifford GM, Smith JS, Plummer M, et al. Human papillomavirus types in invasive cervical cancer worldwide: a meta-analysis. Br J Cancer 2003;88: 63-73.

14 Östör AG. Natural history of cervical intraepithelial neoplasia: a critical review. Int J Gynecol Pathol 1993;12:186-192.

15 Bergeron C, Barrasso R, Beaudenon S, et al. Human papillomaviruses associated with cervical intraepithelial neoplasia. Great diversity and distinct distribution in low-and high-grade lesions. Am J Surg Pathol 1992;16:641-649.

16 de Roda Husman AM, Walboomers JMM, Meijer CJLM, et al. Analysis of cytomorphologically abnormal cervical scrapes for the presence of 27 mucosotropic human papillomavirus genotypes using polymerase chain reaction. Int J Cancer 1994;56:802-806.

17 Gravitt PE, Peyton CL, Apple RJ, et al. Genotyping of 27 human papillomavirus types by using L1 consensus PCR products by a single-hybridization, reverse line blot detection method. J Clin Microbiol 1998;36: 3020-3027.

18 Gravitt PE, Peyton CL, Alessi TQ, et al. Improved amplification of genital human papillomaviruses. J Clin Microbiol 2000;38:357-361.

19 Zuna RE, Moore W, Dunn ST. HPV DNA testing of the residual sample of liquid-based Pap test: utility as a quality assurance monitor. Mod Pathol 2001;14: 147-151.

20 Kurman R, Solomon DS. The Bethesda System for Reporting Cervical/Vaginal Cytologic Diagnoses. Springer-Verlag: New York, 1993.

21 Douglas KL. ThinPrep ${ }^{\mathbb{R}}$ Morphology Reference Manual. Cytyc Corporation: Boxborough, MA, 1994.

22 Scully RE, Bonfiglio TA, Kurman RJ (eds). Classification of Female Genital Tract Tumors, 2nd edn. Springer-Verlag: Berlin, 1994.

23 Kalantari M, Karlsen F, Johansson B, et al. Human papillomavirus findings in relation to cervical intraepithelial neoplasia grade: a study on 476 Stockholm women, using PCR for detection and typing of HPV. Hum Pathol 1997;28:899-904.

24 Clifford GM, Smith JS, Aguado, et al. Comparison of HPV type distribution in high grade lesions and cervical cancer: a meta-analysis. Br J Cancer 2003;89: 101-105.

25 Solomon D, Davey D, Kurman R, et al. The 2001 Bethesda system: terminology for reporting results of cervical cytology. JAMA 2002;287:2114-2119.

26 Wright Jr TC, Cox JT, Massad LS, et al. ASCCPsponsored consensus conference: 2001 consensus guidelines for the management of women with cervical cytological abnormalities. JAMA 2002;287:2120-2129.

27 Kiviat NB, Critchlow CW, Kurman RJ. Reassessment of the morphological continuum of cervical intraepithelial lesions: does it reflect different stages in the progression to cervical carcinoma? In: Muñoz N, Bosch FX, Shah KV, Meheus A (eds). The Epidemiology of Cervical Cancer and Human Papillomavirus. International Agency for Research on Cancer: Lyon, 1992, pp 59-66.

28 Kiviat NB. Natural history of cervical neoplasia: overview and update. Am J Obstet Gynecol 1996;175: 1099-1104.

29 Wright TC, Kurman RJ. A critical review of the morphologic classification systems of preinvasive lesions of the cervix: the scientific basis of the paradigm. Papillomavirus Rep 1994;5:175-181.

30 Reid R, Greenberg M, Jenson AB, et al. Sexually transmitted papillomaviral infections. I. The anatomic distribution and pathologic grade of neoplastic lesions associated with different viral types. Am J Obstet Gynecol 1987;156:212-222.

31 Kurman RJ, Schiffman MH, Lancaster WD, et al. Analysis of individual human papillomavirus types in cervical neoplasia: a possible role for type 18 in rapid progression. Am J Obstet Gynecol 1988;159: 293-296.

32 Wilczynski SP, Bergen S, Walker J, et al. Human papillomaviruses and cervical cancer: analysis of histopathologic features associated with different viral types. Hum Pathol 1988;19:697-704.

33 Lungu O, Sun XW, Felix J, et al. Relationship of human papillomavirus type to grade of cervical intraepithelial neoplasia. JAMA 1992;267:2493-2496.

34 Klinkhamer PJJM, Vooijs GP, de Haan AFJ. Intraobserver and interobserver variability in the diagnosis of epithelial abnormalities in cervical smears. Acta Cytol 1988;32:794-800.

35 Young NA, Naryshkin S, Atkinson BF, et al. Interobserver variability of cervical smears with squamouscell abnormalities: a Philadelphia study. Diagn Cytopathol 1994;11:352-357.

36 Woodhouse SL, Stastny JF, Styer PE, et al. Interobserver variability in subclassification of squamous intra epithelial lesions: results of the College of American Pathologists interlaboratory comparison program in cervicovaginal cytology. Arch Pathol Lab Med 1999; 123:1079-1084.

37 Ismail SM, Colclough AB, Dinnen JS, et al. Reporting cervical intra-epithelial neoplasia (CIN): intra- and interpathologist variation and factors associated with disagreement. Histopathology 1990;16:371-376.

38 Stoler MH, Schiffman. Interobserver reproducibility of cervical cytologic and histologic interpretations: realistic estimates form the ASCUS-LSIL Triage Study. JAMA 2001;285:1500-1505. 